\title{
Five Decades of Hippocampal Place Cells and EEG Rhythms in Behaving Rats
}

\author{
- $L$ Laura Lee Colgin \\ Department of Neuroscience, Center for Learning and Memory, University of Texas at Austin, Austin, Texas 78712
}

Over the last 50 years, much has been learned about the physiology and functions of the hippocampus from studies in freely behaving rats. Two relatively early works in the field provided major insights that remain relevant today. Here, I revisit these studies and discuss how our understanding of the hippocampus has evolved over the last several decades.

\section{Introduction}

When I was given the opportunity to write this article describing how our understanding of hippocampal physiology and function has changed since the time of the first annual Society for Neuroscience meeting, I thought a lot about how to approach the topic. I was told to write this from my own perspective, while also focusing on interesting changes that have happened in the field over the last several years. I decided to revisit works written shortly after the time of the first Society for Neuroscience meeting that greatly impacted the hippocampal field and had a major influence on my own work. I will also discuss examples of more recent studies that built upon these foundations to advance our understanding of hippocampal physiology and function. I focus on findings related to two of my favorite areas of hippocampal research: place cells and EEG rhythms. The insights provided by these decades-old works remain highly significant and relevant to our modern understanding of the hippocampus.

\section{Place cells and the enduring influence of The hippocampus as a cognitive map (O'Keefe and Nadel, 1978)}

What was known about the hippocampus in 1969, the year when the first Society for Neuroscience meeting was held? A decade earlier, Scoville and Milner (1957) had published their groundbreaking report that assessed human patients who had hippocampal damage after undergoing neurosurgery to remove parts of their medial temporal lobes. The amnesia observed in these patients, including patient H.M., for events experienced after their surgeries led researchers to conclude that the primary function of the hippocampus is episodic memory. Before this work, other theories of hippocampal function had been put forward, including hypotheses that the hippocampus is involved in emotion, olfaction, and attention (Andersen et al., 2007).

In 1969, John O'Keefe and colleagues described an important technical breakthrough that would allow future studies to directly relate activity of neurons to animals' behavior and performance on behavioral tasks.

Received April 1, 2019; revised Aug. 19, 2019; accepted Aug. 22, 2019.

This work was supported by National Science Foundation Career Award 1453756, National Institutes of Health Award R01MH102450, and Department of Defense Congressionally Directed Medical Research Program Award W81XWH1810314. The author thanks Ernie Hwaun for help with preparation of Figure 3.

The authors declare no competing financial interests.

Correspondence should be addressed to Laura Lee Colgin at colgin@mail.clm.utexas.edu.

https://doi.org/10.1523/JNEUROSCI.0741-19.2019

Copyright $\odot 2020$ the authors
Although electrodes were placed in the medulla in the original report (Ainsworth et al., 1969), it did not take long for O'Keefe and others to set their sights on the hippocampus. In a subsequent study, O'Keefe and Dostrovsky (1971) implanted electrodes in the hippocampus to record how hippocampal spiking activity was affected by different behaviors (e.g., walking, orienting, sniffing, sleeping, etc.) and presentation of various sensory stimuli. In this initial short report, they described 8 putative neurons in the dorsal hippocampus that fired at particular locations in the testing environment and hypothesized that a function of the hippocampus is to create and store a map of space. Five years later, O'Keefe (1976) published a more thorough study reporting that place cells, neurons in the hippocampus that fire when a rat moves through a particular location in the environment, are a major cell type in the hippocampus. This paper was shortly followed by the publication of a book that has been called "a bible in the study of spatial coding in the hippocampal formation" (Moser et al., 2017), The hippocampus as a cognitive map (O'Keefe and Nadel, 1978).

The Introduction of this highly influential book states the work's purpose: "We shall argue that the hippocampus is the core of a neural memory system providing an objective spatial framework within which the items and events of an organism's experience are located and interrelated" (O'Keefe and Nadel, 1978). This viewpoint remains widely held today (Lisman et al., 2017), at least for many nonhuman species, and the finding that place cells fire in specific spatial locations in a given environment has been replicated by numerous laboratories.

Our understanding of place cells has since expanded to include findings showing that hippocampal "complex-spike cells," the term used at the time to describe putative pyramidal cells, also represent many nonspatial aspects of experience. Initial influential studies from the late Bob Muller, his long-time collaborator John Kubie, and other colleagues showed that changing the stimuli associated with an environment (e.g., color, shape, light vs darkness) could change the firing patterns of place cells (Muller and Kubie, 1987; Quirk et al., 1990; Bostock et al., 1991). In 1999, Emma Wood, Paul Dudchenko, and the late Howard Eichenbaum published a groundbreaking study recording responses of complex spike cells in rats performing an olfactory memory task (Wood et al., 1999). They found that cells in hippocampal subregions CA3 and CA1 responded selectively to salient, nonspatial aspects of the animal's experience, including odor and trial condition (i.e., match or nonmatch). Since then, subsequent studies have shown that place cells represent tactile (Gener et al., 2013), auditory (Aronov et al., 2017), and taste (Herzog et al., 2019) information.

Yet, place cells are not simply computing a conjunctive response to multimodal sensory stimuli. Various lines of evidence indicate that place cell responses change according to animals' past experiences. Place cells 

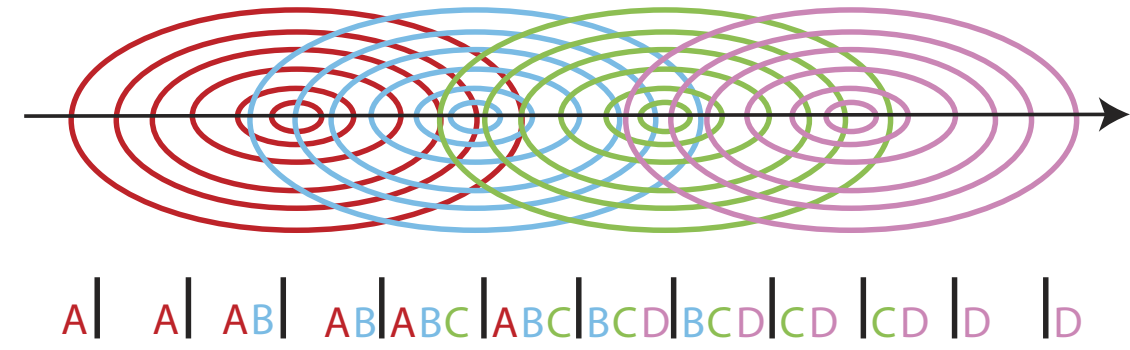

Figure 1. Sequences of place cells firing within individual theta cycles are depicted in a schematic. Four place cells with partially overlapping place fields are depicted in a rat who is running to the right. Individual theta cycles are separated by black horizontal lines. Note how organized place cells fire across successive theta cycles as the rat runs through their place fields. Theta sequences are apparent in the middle theta cycles, with place cells having place fields at earlier and later locations firing at earlier and later theta phases, respectively. Reproduced with permission from Skaggs et al. (1996).

were shown to change their firing patterns in specific environments in which fear conditioning occurred (Moita et al., 2004). However, experiences do not have to be aversive to significantly change place cell activity. In an experiment by Jill Leutgeb and colleagues (2005), place cells in rats were recorded across successive sessions in an environment that was gradually morphed from a square to a circle, and vice versa, through a series of intermediate shapes. Place cell firing patterns in intermediate shapes more closely resembled firing patterns in the square or circle when the square or circle, respectively, was presented first in the sequence (Leutgeb et al., 2005). Moreover, in a follow-up study, place cell firing patterns were found to gradually change across intermediate morph shapes in rats that had been pretrained in a constant location. In contrast, in rats that had been previously trained in square and circle environments placed in separate locations, place cell firing patterns in square-like intermediate shapes remained similar to those in the square and abruptly changed to resemble firing patterns in the circle when animals were placed in circle-like intermediate environments (Colgin et al., 2010). Place cells' firing rates also change according to where an animal was in the recent past or where an animal is heading in the future (Frank et al., 2000; Wood et al., 2000; Ferbinteanu and Shapiro, 2003). Moreover, place cell firing becomes more anticipatory with experience. That is, place cells start firing earlier and earlier before the center of their place fields as animals gain experience in an environment (Mehta et al., 1997, 2000; Ekstrom et al., 2001; Lee et al., 2004; Roth et al., 2012; Gereke et al., 2018).

More recently, the principal cells of the hippocampus have also been shown to represent time. In an initial important study, rats were trained to run in a running wheel for a fixed amount of time during the delay period of a delayed alternation memory task (Pastalkova et al., 2008). Remarkably, hippocampal cells were found to reliably fire at specific times during the delay period, suggesting that hippocampal neurons represent temporal aspects of episodic memories. Subsequent findings from other groups supported the existence of these hippocampal "time cells" (Gill et al., 2011; MacDonald et al., 2011) and further demonstrated that cells respond to the passage of time rather than merely reflecting accumulation of path integration cues during movement (Kraus et al., 2013). In numerous other studies involving random foraging or other behaviors that do not involve learning of specific time intervals, hippocampal cell firing patterns have been shown to remain relatively stable across times similar to the delays used in the aforementioned time cell studies. Thus, it is possible that hippocampal neurons represent time intervals in this way only when time is a salient factor.

Other results support the idea that hippocampal neurons preferentially represent salient aspects of an experience. Hippocampal place cells have been shown to preferentially fire at goal or reward locations. In an initial important study, the proportion of place cells that had place fields in the segment of an annular water maze that contained the escape platform was significantly higher than the proportion of place cells with fields in other maze segments (Hollup et al., 2001). A subsequent important study revealed that this overrepresentation of goal locations developed gradually as animals learned new goal locations and that memory perforpublication. mance was correlated with the proportion of place cells representing goal locations (Dupret et al., 2010). A recent study using calcium imaging of hippocampal neurons in virtual reality provided evidence suggesting that the hippocampus contains a subset of cells that are specialized for representing reward information regardless of spatial location (Gauthier and Tank, 2018).

The flexibility in place cells' firing patterns described above raises questions regarding the extent to which place cells' maps of space remain stable in unchanged environments. The traditional viewpoint is that place cells maintain stable maps in unchanging environments and that this is necessary for place cells to provide a cognitive map (Thompson and Best, 1990). However, recent studies have begun to challenge this view. In hippocampal subregion CA2, place cells' firing patterns change over the time course of hours in constant spatial environments (Mankin et al., 2015). Another study measuring large numbers of CA1 place cells simultaneously in freely behaving mice reported that the specific ensemble of place cells representing a particular environment changed across days when environmental conditions were held constant (Ziv et al., 2013). From day to day, a mixed pattern was observed, with some cells maintaining relatively stable firing patterns from one day to the next and others changing their firing patterns. However, another recent study involving calcium imaging of place cells in freely behaving mice suggested that much of the instability observed in earlier studies may reflect a coherent reorientation of place cell maps to a different environmental landmark than the landmark used to orient the maps in earlier sessions (Kinsky et al., 2018). Thus, the question of whether place fields remain sufficiently stable to provide a stable spatial framework for long-term memories deserves further attention.

The above-discussed studies involved recordings of hippocampal neurons during active waking behaviors. Yet, The hippocampus as a cognitive map also provided the following observation: "For some place units the rat's location in an environment is a necessary, but not sufficient, condition for unit firing; in addition, the animal must be behaving in a specific way. .. " (O'Keefe and Nadel, 1978, p 197). Place cells fire in their place fields during theta-related behaviors (e.g., running) but do not necessarily fire in their place fields when an animal is quietly resting or sleeping, times when the hippocampal network is in the sharp wave-ripple mode. Our understanding of hippocampal physiology and function has been greatly informed by studying specific EEG patterns of the hippocampal network that correlate with different behavioral states and distinct neuronal firing patterns. The next section revisits a seminal work on hippocampal EEG rhythms (Buzsáki et al., 1983) and discusses how our modern understanding of these rhythms has evolved since the time of its

\section{Hippocampal rhythms and the lasting impact of Cellular bases of hippocampal EEG in the behaving rat} (Buzsáki et al., 1983)

The prominent and strikingly sinusoidal $\sim 6-12 \mathrm{~Hz}$ theta rhythm of the hippocampus was first discovered in the hippocampal EEG of rabbits (Jung and Kornmuller, 1938). Over the next few decades, other researchers recorded theta rhythms across different species and suggested a variety of functions for theta, including general arousal (Green and Arduini, 1954) and various aspects of learning (Grastyan et al., 1959; Adey et al., 1960). However, there was little agreement in the field regarding its potential functional relevance, and no study had yet related theta rhythms to cellular activity patterns in the hippocampus.

In 1969, the year of the first Society for Neuroscience meeting, Case Vanderwolf published an important paper describing the major EEG patterns observed in the hippocampus of freely behaving rats (Vanderwolf, 1969). In addition to the aforementioned theta rhythms (also referred to at that time as rhythmical slow activity), he reported two other electrical activity patterns: large-amplitude irregular activity and small- 
a Lap 1

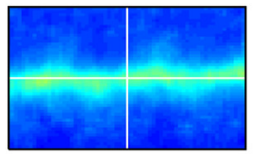

Lap 6

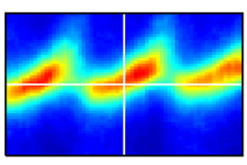

Lap 11

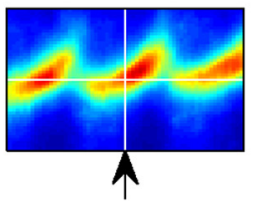

Lap 2

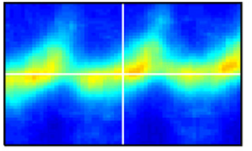

Lap 7

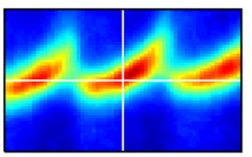

Lap 12

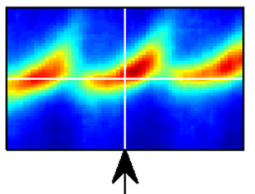

Lap 3

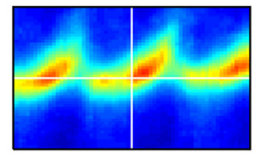

Lap 8

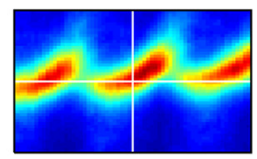

Lap 13

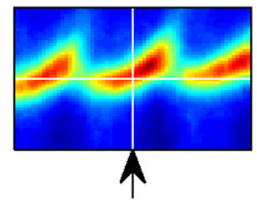

Lap 4

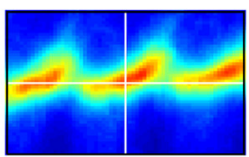

Lap 9

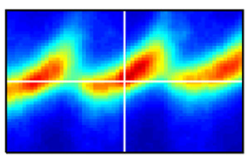

Lap 14

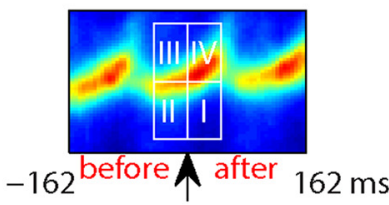

Lap 5

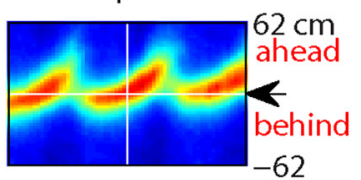

Lap 10

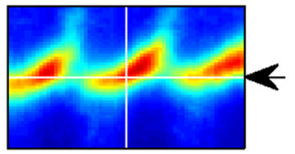

b
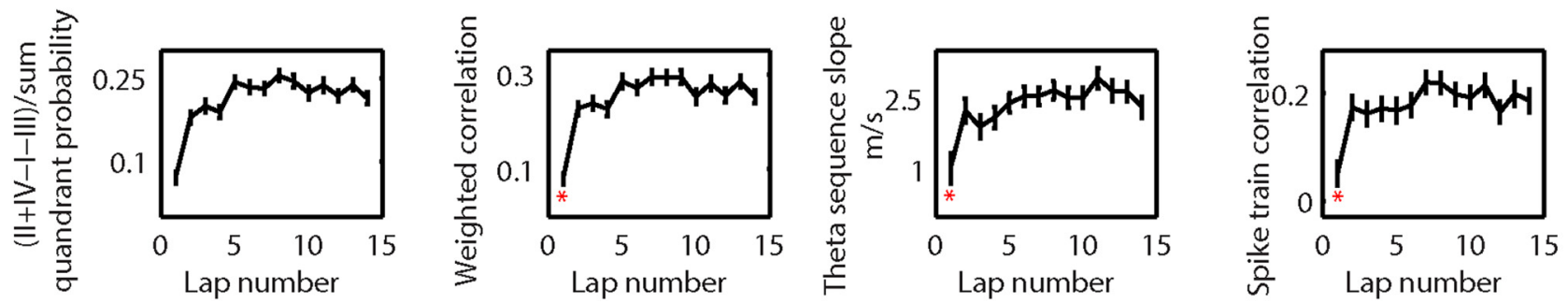

Figure 2. Theta sequences emerge across laps on a novel track. $\boldsymbol{a}$, Averaged decoded probabilities (color-coded) over position ( $y$ axis), centered by the animal's current running position. The $x$ axis indicates time, centered according to the mid-time point of the theta sequence for each lap. $\boldsymbol{b}$, The structure of theta sequences changed significantly between the first lap and subsequent laps (significance indicated by red asterisk). First panel, Probability of (II + IV - I - III)/(I + II + III + IV) quadrant in a $100 \mathrm{~cm}$ and half theta cycle window centered by the animal's current running position and the mid-time point of theta sequence on different laps. Second panel, Weighted correlations between time and decoded positions across laps. Third panel, Theta sequence slopes across laps. Fourth panel, Spike train correlations across laps. Reproduced with permission from Feng et al. (2015).

amplitude irregular activity. The latter pattern was not described in much detail, but a detailed description of specific behaviors associated with theta rhythms and large-amplitude irregular activity was provided. Theta rhythms were reported to occur during active voluntary movements, such as walking and rearing. Large-amplitude irregular activity was observed during "automatic" behaviors, such as licking and chewing. Vanderwolf hypothesized that the different patterns were associated with different cellular circuitry. However, this initial report included no recordings of spiking activity from putative single cells in conjunction with the EEG patterns.

Over a decade later, Vanderwolf and his colleagues Buzsáki and Leung published their seminal paper "Cellular bases of hippocampal EEG in the behaving rat" (Buzsáki et al., 1983). This work again described the major EEG patterns of the hippocampal network but, in addition, investigated their relationship to spiking activity. The paper first described two of the same EEG patterns that had been studied in Vanderwolf's earlier work: theta rhythms and large-amplitude irregular activity. In addition, another pattern referred to then as "hippocampal fast activity $(25-70 \mathrm{~Hz})$," and today known as gamma rhythms, was studied. This important paper revealed many key properties of these rhythms and greatly informed our modern understanding of their origins and functions. The authors of this work explained how the firing patterns of putative pyramidal cells and interneurons correlated with the different rhythms. The paper again reported the different behavioral correlates of theta rhythms and sharp wave-ripples, which were consistent with Vanderwolf's earlier observations (Vanderwolf, 1969). The authors further reported that gamma occurs under all behavioral conditions. Below, I discuss some of the key findings reported in this seminal study of hippocampal EEG patterns and spiking activity and then discuss more recent studies that built upon this fundamental knowledge.
Rhythmical slow activity," more commonly known now as the theta rhythm. Buzsáki et al. (1983) wrote, "The most characteristic EEG type of the hippocampus is its rhythmical slow activity (theta pattern), which is a high amplitude regular EEG pattern occurring particularly in nonprimate mammals." The authors further reported that multiple complex spike cells fire at a specific phase of the theta cycle followed by a suppression of firing at the approximately opposite phase, the phase at which interneurons preferentially fire. We now know that these windows of time within theta cycles between inhibitory events allow ensembles of place cells to fire in organized sequences (Skaggs et al., 1996; Dragoi and Buzsáki, 2006; Foster and Wilson, 2007). These theta-organized ensembles are commonly referred to as "theta sequences." As a rat moves through an individual place cell's place field, the place cell spikes across several successive theta cycles, initially firing at late theta phases during the first theta cycles and then at progressively earlier theta phases on later theta cycles (O'Keefe and Recce, 1993; Skaggs et al., 1996). When multiple place cells become linked to each other as an animal learns a specific trajectory, place cells with consecutively occurring place fields will be activated in an orderly fashion within individual theta cycles, with cells representing just visited and upcoming locations firing at early and late theta phases, respectively (Fig. 1). It is possible that such coordinated theta sequences of cells, rather than individual place cells, play a key role in hippocampal memory operations during active behaviors. In line with this idea, theta sequences are not immediately apparent when a rat first encounters an environment but instead develop with experience (Feng et al., 2015) (Fig. 2). Also, it has recently been reported that theta sequences do not fully develop in the rat hippocampus until around postnatal day 23 , although individual place fields are already present at earlier ages (Farooq and Dragoi, 2019; Muessig et al., 2019). Intriguingly, this devel- 


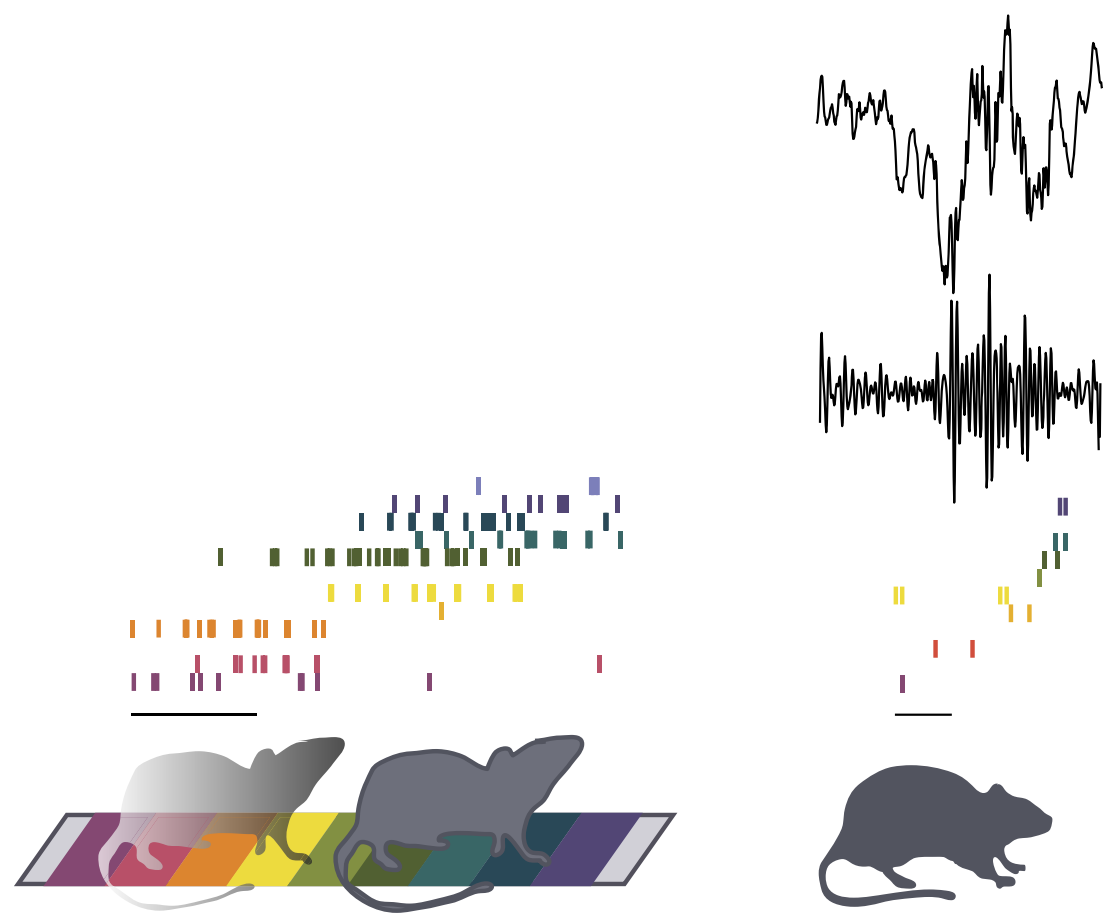

Figure 3. Hippocampal replay during sharp wave-ripples. Left, Each row of colored tick marks indicates spikes from a place cell. The ensemble of place cells is activated sequentially as a rat passes through its place fields during active waking behavior. Calibration: 500 ms. Right, An example sharp wave-ripple (top) recorded as the rat subsequently rested; a bandpass filtered $(150-300 \mathrm{~Hz})$ version of the sharp wave-ripple is shown below. Spikes from the same place cell ensemble on the left are replayed at a faster time scale during the sharp wave-ripple. Calibration: $50 \mathrm{~ms}$. Reproduced in a modified form with permission from Colgin (2016).

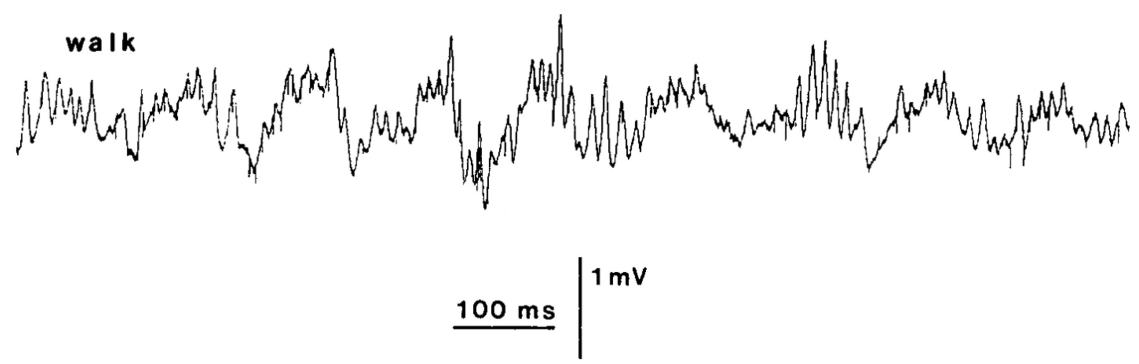

ga m ma - thet a

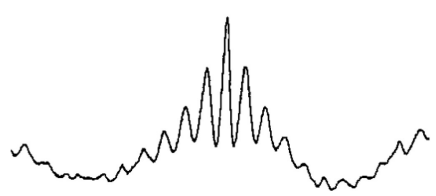

Figure 4. Theta-modulated gamma rhythms. Top, A recording of theta-gamma rhythms from the hippocampus of an actively moving rat. The gamma rhythms that are superimposed on theta rhythms wax and wane within theta cycles. Bottom, A gammatriggered average of a hippocampal recording from a freely moving rat. Gamma activity is highest in magnitude at a particular part of the theta cycle, namely, the theta peak. Reproduced with permission from Bragin et al. (1995).

opmental stage coincides with the emergence of long-term memory retention in rats (Alberini and Travaglia, 2017).

Accumulating recent work suggests that theta sequences may be transmitted to areas downstream of the hippocampus, such as the PFC, to direct rats' future behaviors. An initial important study showed that place cell ensembles alternated between representing two sequences of locations that corresponded to the two maze arms that an animal was deciding between at a choice point in a spatial decision task (Johnson and Redish, 2007). A subsequent study showed that sequences of place cells represented longer trajectories extending ahead of the current position as animals headed to goal locations that were further away and represented shorter trajectories when animals were headed to nearby goal locations (Wikenheiser and Redish, 2015). A recent study showed that hippocampal and PFC sequences during theta represent the same spatial trajectories during a spatial alternation task (Zielinski et al., 2019), in line with the possibility that transmission of theta sequences from the hippocampus to the PFC facilitates memoryguided behaviors and decision-making. Sequences of place cells that were activated during theta are subsequently reactivated during sharp wave-ripples occurring in sleep and rest, perhaps strengthening connections between the hippocampus and PFC, as described in the next section.

"Large-amplitude irregular waves," today more commonly known as sharp wave-ripples. Sharp wave-ripples are irregularly occurring waves of $\sim 40-100 \mathrm{~ms}$ in duration with superimposed $\sim 150-250 \mathrm{~Hz}$ ripple waves (Buzsáki, 1986; Kudrimoti et al., 1999; Nádasdy et al., 1999). Buzsáki et al. (1983) reported that bursts of spikes from several complex spike cells occurred during sharp waves. We now know that these bursts of activity correspond to temporally compressed reactivation of place cell activity patterns that occurred during earlier active waking behaviors (Wilson and McNaughton, 1994; Kudrimoti et al., 1999; Nádasdy et al., 1999; Lee and Wilson, 2002) (Fig. 3). This reactivation has been hypothesized to allow the hippocampus to transmit compressed versions of experiences initially stored in the hippocampus to neocortical outputs as part of the memory consolidation process during sleep. Evidence for this hypothesis was first provided by a study showing that pairwise correlations between place cells during active waking behaviors were reactivated during sharp wave-ripples in subsequent sleep (Wilson and McNaughton, 1994). Later studies extended these findings to show that place cells that fired sequentially during waking behaviors were later replayed in the same order during sharp wave-ripples in sleep (Nádasdy et al., 1999; Lee and Wilson, 2002). Other studies showed that disruption of sharp wave-ripples during sleep impaired later memory performance (Girardeau et al., 2009; Ego-Stengel and Wilson, 2010), providing further important support for the memory consolidation hypothesis. Moreover, activity patterns of PFC neurons during wakefulness were found to reactivate during sleep at times when sharp waveripples occurred in the hippocampus (Peyrache et al., 2009). This finding lent further support to the idea that sharp wave-rippleassociated reactivation serves to transmit memories that were initially stored in the hippocampus to the neocortex during memory consolidation.

As Buzsáki et al. (1983) pointed out, though, sharp wave-ripples also occur during awake behaviors, such as "drinking, quiet sitting, and grooming." Many recent studies have supported the hypothesis that sharp wave-ripple-associated replay that occurs during waking behaviors is also functionally important. Disrupting awake sharp wave-ripples as animals learned a spatial memory task was found to impair memory 
performance (Jadhav et al., 2012). Awake replay events can represent spatially remote trajectories (Davidson et al., 2009; Karlsson and Frank, 2009) or even trajectories that have never been experienced (Gupta et al., 2010), but they tend to represent trajectories that begin close to an animal's current location (Davidson et al., 2009; Karlsson and Frank, 2009). These findings led researchers to suggest that awake replay may be involved in retrieval of representations of upcoming trajectories during planning of future paths and deliberating between potential future paths. In support of this hypothesis, replay during awake sharp wave-ripples was found to represent paths that rats subsequently traversed to reach a learned goal location (Pfeiffer and Foster, 2013). Remarkably, awake replay was later found to also represent paths toward locations where rats had previously been shocked, suggesting that awake replay is also involved in retrieval of memories of paths to be avoided (Wu et al., 2017).

We also now know that awake replay occurs in both forward and reverse order (Foster and Wilson, 2006; Diba and Buzsáki, 2007). Forward replay corresponds to sequences of place cell spikes that are reactivated during sleep and rest in the same order as occurred during earlier active behaviors. Forward replay often occurs before rats initiate a trajectory, suggesting that forward replay during awake behaviors plays a role in planning (Diba and Buzsáki, 2007). Reverse replay refers to sequences of place cells that are reactivated but in an order that is opposite to their earlier order of activation during active behaviors. Reverse replay is likely to occur at the end of a run (Diba and Buzsáki, 2007), consistent with reverse replay serving a function that relates to experience in the recent past rather than the future. A recent study revealed that the rate of reverse replay events, but not forward replay events, during awake sharp waveripples increases with increasing amounts of reward (Ambrose et al., 2016). This result is consistent with the hypothesis that reverse replay plays a role in learning paths toward rewards.

Fast activity, also known as gamma rhythms. Buzsáki et al. (1983) also provided key insights about gamma rhythms. First, they reported that "the power of fast activity was higher during walking and running compared with the power obtained during immobility and drinking." Walking and running are behaviors associated with theta rhythms, and it was subsequently shown that not only is gamma power highest during thetarelated behaviors but also that gamma power is maximal at a particular portion of the theta cycle (Soltesz and Deschênes, 1993; Bragin et al., 1995; Csicsvari et al., 2003) (Fig. 4).

What functions could be served by this theta-gamma coupling? Pioneering work by the late John Lisman provides potential answers to this question (for review, see Lisman and Jensen, 2013). Lisman hypothesized that different gamma cycles within a theta cycle could provide a substrate for ordering sequences of items within a memory. He further suggested that pauses in neuronal firing between separate items are critical for accurate downstream readout of a memory. Gamma rhythms are thought to provide such pauses in neuronal firing between sequentially represented items in a memory. Specifically, hippocampal gamma rhythms reflect high-frequency rhythmic firing of interneurons, as was first reported by Buzsáki and colleagues in their seminal 1983 paper (Buzsáki et al., 1983). This finding was later corroborated by intracellular recordings from hippocampal pyramidal cells that revealed IPSPs occurring at gamma frequency at times when gamma rhythms were observed in the hippocampal EEG (Soltesz and Deschênes, 1993). These results are consistent with recordings of place cell firing during gamma rhythms in behaving animals. Place cells do not fire continuously at high enough rates to sustain a burst of gamma cycles occurring within a theta cycle. Instead, each place cell may only fire in one gamma cycle, or across only a few gamma cycles, within each theta cycle (for examples, see Zheng et al., 2016, their Fig. 3).

In 1983, Buzsáki et al. (1983) also noted that urethane anesthesia decreased the power of higher-frequency gamma rhythms while increasing the power of gamma rhythms in the $25-50 \mathrm{~Hz}$ range. Little was made of this observation at the time, but it is consistent with more recent work that has revealed different frequencies of gamma rhythms driven by different hippocampal inputs. Urethane anesthesia suppresses excitatory transmission from perforant path inputs to the hippocampus from the entorhinal cortex (Ylinen et al., 1995), and higher-frequency "fast" $(\sim 65-95 \mathrm{~Hz})$ gamma rhythms have been linked to fast gamma inputs from medial entorhinal cortex (Colgin et al., 2009; Kemere et al., 2013; Schomburg et al., 2014). In contrast, "slow" ( $25-50 \mathrm{~Hz})$ gamma rhythms are driven by intrahippocampal inputs (Colgin et al., 2009; Kemere et al., 2013; Schomburg et al., 2014; Hsiao et al., 2016).

Researchers have hypothesized that slow and fast gamma rhythms serve different functions, but this hypothesis requires further testing. Although recent studies have provided clues about potential slow and fast gamma functions, there are also differences across studies that are difficult to interpret. An example that demonstrates this point is provided by studies investigating gamma rhythms in rodents performing delayed spatial alternation tasks. A study in mice reported that fast gamma, but not slow gamma, power was increased on the central arm of the task (Yamamoto et al., 2014). The authors further showed that silencing medial entorhinal cortex inputs to the hippocampus on the central arm suppressed fast gamma and hindered memory performance. Based on these results, the authors concluded that fast gamma rhythms are important for working memory. However, another study in rats reported that, while both slow $(\sim 40 \mathrm{~Hz})$ and fast $(\sim 80 \mathrm{~Hz})$ gamma were increased on the center arm of a similar task, slow gamma was more strongly increased than fast gamma (Schomburg et al., 2014). The authors of this study assumed that the central arm portion of this task is associated with memory recall and suggested that slow and fast gamma work in cooperation to facilitate memory recall on the central arm. A more recent study (Fernández-Ruiz et al., 2017), again using a similar behavioral task, reported that place cell spikes were more strongly phase-locked to slow gamma than to fast gamma on the central arm. This study interpreted this result as reflecting enhanced input to CA1 from CA3 during memory recall. Although these and other studies provided important insights regarding the potential roles of slow and fast gamma rhythms in memory (for review, see Colgin, 2015), more work is needed to better understand whether slow and fast gamma play unique roles in memory performance.

In conclusion, since the time of the first annual Society for Neuroscience meeting, the small sample of studies highlighted above, together with many other studies that have not been mentioned, have greatly impacted our understanding of the physiology and function of the hippocampus. Much has been learned over the last few decades from investigations of inputs to the hippocampus (e.g., the discovery of spatially modulated "grid cells" in the superficial layers of the medial entorhinal cortex that project to the hippocampus) (Hafting et al., 2005). How hippocampal computations affect downstream structures to facilitate memory-guided behaviors remains more of a mystery. Over the next decade, it will be exciting to see whether real-time manipulation of theta sequences and sharp wave-associated replay (Ciliberti et al., 2018; Hu et al., 2018) can direct animals' future trajectories and decisions in memory-guided tasks.

\section{References}

Adey WR, Dunlop CW, Hendrix CE (1960) Hippocampal slow waves: distribution and phase relationships in the course of approach learning. Arch Neurol 3:74-90.

Ainsworth A, Gaffan GD, O'Keefe J, Sampson R (1969) A technique for recording units in the medulla of the awake, freely moving rat. J Physiol 202:80-82

Alberini CM, Travaglia A (2017) Infantile amnesia: a critical period of learning to learn and remember. J Neurosci 37:5783-5795.

Ambrose RE, Pfeiffer BE, Foster DJ (2016) Reverse replay of hippocampal place cells is uniquely modulated by changing reward. Neuron 91:11241136.

Andersen P, Morris R, Amaral D, Bliss T, O’Keefe J (2007) The hippocampus book. Oxford: Oxford UP.

Aronov D, Nevers R, Tank DW (2017) Mapping of a non-spatial dimension by the hippocampal-entorhinal circuit. Nature 543:719-722.

Bostock E, Muller RU, Kubie JL (1991) Experience-dependent modifications of hippocampal place cell firing. Hippocampus 1:193-205.

Bragin A, Jandó G, Nádasdy Z, Hetke J, Wise K, Buzsáki G (1995) Gamma $(40-100 \mathrm{~Hz})$ oscillation in the hippocampus of the behaving rat. J Neurosci 15:47-60.

Buzsáki G (1986) Hippocampal sharp waves: their origin and significance. Brain Res 398:242-252. 
Buzsáki G, Leung LW, Vanderwolf CH (1983) Cellular bases of hippocampal EEG in the behaving rat. Brain Res 287:139-171.

Ciliberti D, Michon F, Kloosterman F (2018) Real-time classification of experience-related ensemble spiking patterns for closed-loop applications. Elife 7:e36275.

Colgin LL (2015) Do slow and fast gamma rhythms correspond to distinct functional states in the hippocampal network? Brain Res 1621:309-315.

Colgin LL, Leutgeb S, Jezek K, Leutgeb JK, Moser EI, McNaughton BL, Moser MB (2010) Attractor-map versus autoassociation based attractor dynamics in the hippocampal network. J Neurophysiol 104:35-50.

Colgin LL (2016) Rhythms of the hippocampal network. Nat Rev Neurosci 17:239-249.

Colgin LL, Denninger T, Fyhn M, Hafting T, Bonnevie T, Jensen O, Moser MB, Moser EI (2009) Frequency of gamma oscillations routes flow of information in the hippocampus. Nature 462:353-357.

Csicsvari J, Jamieson B, Wise KD, Buzsáki G (2003) Mechanisms of gamma oscillations in the hippocampus of the behaving rat. Neuron 37:311-322.

Davidson TJ, Kloosterman F, Wilson MA (2009) Hippocampal replay of extended experience. Neuron 63:497-507.

Diba K, Buzsáki G (2007) Forward and reverse hippocampal place-cell sequences during ripples. Nat Neurosci 10:1241-1242.

Dragoi G, Buzsáki G (2006) Temporal encoding of place sequences by hippocampal cell assemblies. Neuron 50:145-157.

Dupret D, O’Neill J, Pleydell-Bouverie B, Csicsvari J (2010) The reorganization and reactivation of hippocampal maps predict spatial memory performance. Nat Neurosci 13:995-1002.

Ego-Stengel V, Wilson MA (2010) Disruption of ripple-associated hippocampal activity during rest impairs spatial learning in the rat. Hippocampus 20:1-10.

Ekstrom AD, Meltzer J, McNaughton BL, Barnes CA (2001) NMDA receptor antagonism blocks experience-dependent expansion of hippocampal "place fields." Neuron 31:631-638.

Farooq U, Dragoi G (2019) Emergence of preconfigured and plastic timecompressed sequences in early postnatal development. Science 363:168173.

Feng T, Silva D, Foster DJ (2015) Dissociation between the experiencedependent development of hippocampal theta sequences and single-trial phase precession. J Neurosci 35:4890-4902.

Ferbinteanu J, Shapiro ML (2003) Prospective and retrospective memory coding in the hippocampus. Neuron 40:1227-1239.

Fernández-Ruiz A, Oliva A, Nagy GA, Maurer AP, Berényi A, Buzsáki G (2017) Entorhinal-CA3 dual-input control of spike timing in the hippocampus by theta-gamma coupling. Neuron 93:1213-1226.e5.

Foster DJ, Wilson MA (2006) Reverse replay of behavioural sequences in hippocampal place cells during the awake state. Nature 440:680-683.

Foster DJ, Wilson MA (2007) Hippocampal theta sequences. Hippocampus 17:1093-1099.

Frank LM, Brown EN, Wilson M (2000) Trajectory encoding in the hippocampus and entorhinal cortex. Neuron 27:169-178.

Gauthier JL, Tank DW (2018) A dedicated population for reward coding in the hippocampus. Neuron 99:179-193.e7.

Gener T, Perez-Mendez L, Sanchez-Vives MV (2013) Tactile modulation of hippocampal place fields. Hippocampus 23:1453-1462.

Gereke BJ, Mably AJ, Colgin LL (2018) Experience-dependent trends in CA1 theta and slow gamma rhythms in freely behaving mice. J Neurophysiol 119:476-489.

Gill PR, Mizumori SJ, Smith DM (2011) Hippocampal episode fields develop with learning. Hippocampus 21:1240-1249.

Girardeau G, Benchenane K, Wiener SI, Buzsáki G, Zugaro MB (2009) Selective suppression of hippocampal ripples impairs spatial memory. Nat Neurosci 12:1222-1223.

Grastyan E, Lissak K, Madarasz I, Donhoffer H (1959) Hippocampal electrical activity during the development of conditioned reflexes. Electroencephalogr Clin Neurophysiol 11:409-430.

Green JD, Arduini AA (1954) Hippocampal electrical activity in arousal. J Neurophysiol 17:533-557.

Gupta AS, van der Meer MA, Touretzky DS, Redish AD (2010) Hippocampal replay is not a simple function of experience. Neuron 65:695-705.

Hafting T, Fyhn M, Molden S, Moser MB, Moser EI (2005) Microstructure of a spatial map in the entorhinal cortex. Nature 436:801-806.

Herzog LE, Pascual LM, Scott SJ, Mathieson ER, Katz DB, Jadhav SP (2019)
Interaction of taste and place coding in the hippocampus. J Neurosci 39:3057-3069.

Hollup SA, Molden S, Donnett JG, Moser MB, Moser EI (2001) Accumulation of hippocampal place fields at the goal location in an annular watermaze task. J Neurosci 21:1635-1644.

Hsiao YT, Zheng C, Colgin LL (2016) Slow gamma rhythms in CA3 are entrained by slow gamma activity in the dentate gyrus. J Neurophysiol 116:2594-2603

Hu S, Ciliberti D, Grosmark AD, Michon F, Ji D, Penagos H, Buzsáki G, Wilson MA, Kloosterman F, Chen Z (2018) Real-time readout of largescale unsorted neural ensemble place codes. Cell Rep 25:2635-2642.e5.

Jadhav SP, Kemere C, German PW, Frank LM (2012) Awake hippocampal sharp-wave ripples support spatial memory. Science 336:1454-1458.

Johnson A, Redish AD (2007) Neural ensembles in CA3 transiently encode paths forward of the animal at a decision point. J Neurosci 27:1217612189.

Jung R, Kornmuller AE (1938) Eine methodik der ableitung lokalisierter potentialschwankungen aus subcorticalen hirngebieten. Arch Psychiat Nervenkr 109:1-30.

Karlsson MP, Frank LM (2009) Awake replay of remote experiences in the hippocampus. Nat Neurosci 12:913-918.

Kemere C, Carr MF, Karlsson MP, Frank LM (2013) Rapid and continuous modulation of hippocampal network state during exploration of new places. PLoS One 8:e73114.

Kinsky NR, Sullivan DW, Mau W, Hasselmo ME, Eichenbaum HB (2018) Hippocampal place fields maintain a coherent and flexible map across long timescales. Curr Biol 28:3578-3588.e76.

Kraus BJ, Robinson RJ 2nd, White JA, Eichenbaum H, Hasselmo ME (2013) Hippocampal "time cells": time versus path integration. Neuron 78: 1090-1101.

Kudrimoti HS, Barnes CA, McNaughton BL (1999) Reactivation of hippocampal cell assemblies: effects of behavioral state, experience, and EEG dynamics. J Neurosci 19:4090-4101.

Lee AK, Wilson MA (2002) Memory of sequential experience in the hippocampus during slow wave sleep. Neuron 36:1183-1194.

Lee I, Rao G, Knierim JJ (2004) A double dissociation between hippocampal subfields: differential time course of CA3 and CA1 place cells for processing changed environments. Neuron 42:803-815

Leutgeb JK, Leutgeb S, Treves A, Meyer R, Barnes CA, McNaughton BL, Moser MB, Moser EI (2005) Progressive transformation of hippocampal neuronal representations in "morphed" environments. Neuron 48 : 345-358.

Lisman JE, Jensen O (2013) The theta-gamma neural code. Neuron 77: 1002-1016.

Lisman JE, Buzsáki G, Eichenbaum H, Nadel L, Ranganath C, Redish AD (2017) How the hippocampus contributes to memory, navigation and cognition. Nat Neurosci 20:1434-1447.

MacDonald CJ, Lepage KQ, Eden UT, Eichenbaum H (2011) Hippocampal "time cells" bridge the gap in memory for discontiguous events. Neuron 71:737-749.

Mankin EA, Diehl GW, Sparks FT, Leutgeb S, Leutgeb JK (2015) Hippocampal CA2 activity patterns change over time to a larger extent than between spatial contexts. Neuron 85:190-201.

Mehta MR, Barnes CA, McNaughton BL (1997) Experience-dependent, asymmetric expansion of hippocampal place fields. Proc Natl Acad Sci U S A 94:8918-8921.

Mehta MR, Quirk MC, Wilson MA (2000) Experience-dependent asymmetric shape of hippocampal receptive fields. Neuron 25:707-715.

Moita MA, Rosis S, Zhou Y, LeDoux JE, Blair HT (2004) Putting fear in its place: remapping of hippocampal place cells during fear conditioning. J Neurosci 24:7015-7023.

Moser EI, Moser MB, McNaughton BL (2017) Spatial representation in the hippocampal formation: a history. Nat Neurosci 20:1448-1464.

Muessig L, Lasek M, Varsavsky I, Cacucci F, Wills TJ (2019) Coordinated emergence of hippocampal replay and theta sequences during post-natal development. Curr Biol 29:834-840.e4.

Muller RU, Kubie JL (1987) The effects of changes in the environment on the spatial firing of hippocampal complex-spike cells. J Neurosci 7:19511968.

Nádasdy Z, Hirase H, Czurkó A, Csicsvari J, Buzsáki G (1999) Replay and time compression of recurring spike sequences in the hippocampus. J Neurosci 19:9497-9507. 
O'Keefe J (1976) Place units in the hippocampus of the freely moving rat. Exp Neurol 51:78-109.

O'Keefe J, Dostrovsky J (1971) The hippocampus as a spatial map: preliminary evidence from unit activity in the freely-moving rat. Brain Res 34:171-175.

O'Keefe J, Nadel L (1978) The hippocampus as a cognitive map. New York: Oxford UP.

O'Keefe J, Recce ML (1993) Phase relationship between hippocampal place units and the EEG theta rhythm. Hippocampus 3:317-330.

Pastalkova E, Itskov V, Amarasingham A, Buzsáki G (2008) Internally generated cell assembly sequences in the rat hippocampus. Science 321: $1322-1327$.

Peyrache A, Khamassi M, Benchenane K, Wiener SI, Battaglia FP (2009) Replay of rule-learning related neural patterns in the prefrontal cortex during sleep. Nat Neurosci 12:919-926.

Pfeiffer BE, Foster DJ (2013) Hippocampal place-cell sequences depict future paths to remembered goals. Nature 497:74-79.

Quirk GJ, Muller RU, Kubie JL (1990) The firing of hippocampal place cells in the dark depends on the rat's recent experience. J Neurosci 10: $2008-2017$.

Roth ED, Yu X, Rao G, Knierim JJ (2012) Functional differences in the backward shifts of CA1 and CA3 place fields in novel and familiar environments. PLoS One 7:e36035.

Schomburg EW, Fernández-Ruiz A, Mizuseki K, Berényi A, Anastassiou CA, Koch C, Buzsáki G (2014) Theta phase segregation of input-specific gamma patterns in entorhinal-hippocampal networks. Neuron 84:470-485.

Scoville WB, Milner B (1957) Loss of recent memory after bilateral hippocampal lesions. J Neurol Neurosurg Psychiatry 20:11-21.

Skaggs WE, McNaughton BL, Wilson MA, Barnes CA (1996) Theta phase precession in hippocampal neuronal populations and the compression of temporal sequences. Hippocampus 6:149-172.

Soltesz I, Deschênes M (1993) Low- and high-frequency membrane potential oscillations during theta activity in CA1 and CA3 pyramidal neurons of the rat hippocampus under ketamine-xylazine anesthesia. J Neurophysiol 70:97-116.

Thompson LT, Best PJ (1990) Long-term stability of the place-field activity of single units recorded from the dorsal hippocampus of freely behaving rats. Brain Res 509:299-308.

Vanderwolf CH (1969) Hippocampal electrical activity and voluntary movement in the rat. Electroencephalogr Clin Neurophysiol 26:407-418.

Wikenheiser AM, Redish AD (2015) Hippocampal theta sequences reflect current goals. Nat Neurosci 18:289-294.

Wilson MA, McNaughton BL (1994) Reactivation of hippocampal ensemble memories during sleep. Science 265:676-679.

Wood ER, Dudchenko PA, Eichenbaum H (1999) The global record of memory in hippocampal neuronal activity. Nature 397:613-616.

Wood ER, Dudchenko PA, Robitsek RJ, Eichenbaum H (2000) Hippocampal neurons encode information about different types of memory episodes occurring in the same location. Neuron 27:623-633.

Wu CT, Haggerty D, Kemere C, Ji D (2017) Hippocampal awake replay in fear memory retrieval. Nat Neurosci 20:571-580.

Yamamoto J, Suh J, Takeuchi D, Tonegawa S (2014) Successful execution of working memory linked to synchronized high-frequency gamma oscillations. Cell 157:845-857.

Ylinen A, Soltész I, Bragin A, Penttonen M, Sik A, Buzsáki G (1995) Intracellular correlates of hippocampal theta rhythm in identified pyramidal cells, granule cells, and basket cells. Hippocampus 5:78-90.

Zheng C, Bieri KW, Hsiao YT, Colgin LL (2016) Spatial sequence coding differs during slow and fast gamma rhythms in the hippocampus. Neuron 89:398-408.

Zielinski MC, Shin JD, Jadhav SP (2019) Coherent coding of spatial position mediated by theta oscillations in the hippocampus and prefrontal cortex. J Neurosci 39:4550-4565.

Ziv Y, Burns LD, Cocker ED, Hamel EO, Ghosh KK, Kitch LJ, El Gamal A, Schnitzer MJ (2013) Long-term dynamics of CA1 hippocampal place codes. Nat Neurosci 16:264-266. 